\title{
Competitive Adsorption of Three Human Plasma Proteins onto Sulfhydryl-to-sulfonate Gradient Surfaces ${ }^{\dagger}$
}

\author{
Yong-Xue Ding and Vladimir Hlady* \\ Department of Bioengineering, University of Utah, Salt Lake City, UT 84112, USA
}

RECEIVED JANUARY 5, 2011; REVISED MARCH 21, 2011; ACCEPTED MARCH 29, 2011

\begin{abstract}
Competitive adsorption of three human plasma proteins: albumin (HSA), fibrinogen (Fgn), and immunoglobulin $\mathrm{G}$ ( $\operatorname{IgG}$ ) from their ternary solution mixtures onto a sulfhydryl-to-sulfonate gradient surface was investigated using spatially-resolved total internal reflection fluorescence (TIRF) and autoradiography. The concentration of each protein in the ternary solution mixture was kept at an equivalent of $1 / 100$ of its physiological concentration in blood plasma. The three proteins displayed different adsorption and desorption characteristics. Each protein adsorbed less to the sulfonate region than to the sulfhydryl region of the gradient. The adsorption-desorption kinetics revealed large differences in the adsorption and desorption rates of three proteins. By fitting the experimental data to a simple model of competitive protein adsorption, the affinity of each protein to the surface at the gradient center position was ranked as: Fgn > HSA >> IgG. Competitive exchange of adsorbed proteins was related to the magnitude of desorption rate constants. Such competitive adsorption of the three major human plasma proteins illustrates the complex dynamics of blood proteins - biomaterials interactions.(doi: 10.5562/cca1821)
\end{abstract}

Keywords: surface gradient, fibrinogen, albumin, immunoglobulin G, competitive protein adsorption

\section{INTRODUCTION}

The role of adsorbed proteins in biomaterial-host interactions is one of the canonical themes in the field of biocompatibility. The causality between the state of biomaterial-adsorbed proteins and the subsequent response by the host organism to the biomaterial implants has been firmly established in a number of studies. ${ }^{1-7}$ While numerous experimental protein adsorption studies have been reported in the literature ${ }^{1}$, the majority of these studies were limited to a single protein and/or uniform biomaterial "model" surface. ${ }^{8-14}$ Even for these simplified experiments a number of assumptions like, adsorption into a monolayer, uniform surface energy, or uniform protein-surface affinity, were introduced to reduce the system to a manageable set of parameters.

One way to broaden the scope of protein adsorption experiments is to construct and use non-uniform surfaces of mixed or variable chemistry. Such surfaces serve as a better model for molecular richness typically encountered in biological systems. ${ }^{15,16}$ Among the nonuniform substrates for protein adsorption so-called

\footnotetext{
${ }^{1}$ According to PubMed $\approx 1,000$ papers are published every year on this topic.
}

"gradient" surfaces have gained popularity since their introduction in late $80,{ }^{17-28}$ In our laboratory, we have used the following gradients to study protein adsorption: dichlorodimethylsilyl- and octadecyldimethylsilyl (C18)silica gradients, ${ }^{19-21,24,25}$ grafted PEO-silica gradient, ${ }^{23}$ and quarternary amine-silica gradient; ${ }^{22}$ each prepared by using the methodology originally developed by Elwing. ${ }^{18}$ Elwing's approach to generate surface gradients utilizes two solvents layered on the top of each other with a reactive silane reagent present initially only in the lower solvent. Diffusion of the reagent into the upper solvent creates a silane concentration gradient across the two liquids that controls the surface silanization reaction. However, the diffusion approach suffers from limited control over the surface density, the length and the steepness of the gradient.

Recently we have engineered a more versatile gradient based on an initially uniform 3-mercaptopropylsilane (MTS) monolayer that is selectively oxidized by UV irradiation using a movable mask. ${ }^{27,28}$ In the preceding paper we reported on the adsorption of three human plasma proteins, albumin (HSA), immunoglobulin G (IgG) and fibrinogen (Fgn) onto the sulfhydryl-tosulfonate chemistry gradient prepared by selective UV oxidation of MTS monolayer. In that study the adsorp-

\footnotetext{
$\dagger$ This article belongs to the Special Issue Chemistry of Living Systems devoted to the intersection of chemistry with life.

* Author to whom correspondence should be addressed. (E-mail: vladimir.hlady@utah.edu)
} 
tion was studied from the solutions containing only one of the three proteins. ${ }^{28}$ Here we report on the study in which the adsorption was carried out from the ternary protein solution mixtures containing one labeled protein and other two unlabeled proteins. When such experiments are performed with three ternary solution mixtures, one for each of the three labeled proteins, a composite protein adsorption-desorption picture emerges from which the rate constants for protein-surface and protein-protein interactions could be elucidated. To extract these parameters from the experiments we used a simple model for protein adsorption from a ternary solution mixture that accounted for the competition for the surface sites on which three proteins adsorb independently from each other (Figure 1). The model can be mathematically presented as:

$$
\begin{aligned}
& \frac{\mathrm{d} \theta_{1}(t)}{\mathrm{d} t}=k_{1}\left[1-\theta_{1}(t)-\theta_{2}(t)-\theta_{3}(t)\right] c_{1}(0, t)-r_{1} \theta_{1}(t) \\
& \frac{\mathrm{d} \theta_{2}(t)}{\mathrm{d} t}=k_{2}\left[1-\theta_{1}(t)-\theta_{2}(t)-\theta_{3}(t)\right] c_{2}(0, t)-r_{2} \theta_{2}(t) \\
& \frac{\mathrm{d} \theta_{3}(t)}{\mathrm{d} t}=k_{3}\left[1-\theta_{1}(t)-\theta_{2}(t)-\theta_{3}(t)\right] c_{3}(0, t)-r_{3} \theta_{3}(t)
\end{aligned}
$$

where $c(0, t)$ is the protein concentration right next to the adsorbing surface at time $t, \theta(t)$ is the fraction of the surface covered by a particular protein at time $t, k$ is the adsorption rate constant, $r$ is the desorption rate constant and the subscripts 1,2, and 3 denote the three protein species. Assuming that the adsorption of each protein at saturation results in a full monolayer with the adsorbed amount, $\Gamma^{\max }$, the fraction of the surface covered, $\theta$, can be defined as $\theta_{n}(t)=\Gamma_{n}(t) / \Gamma_{n}^{\max }$, where $\Gamma_{n}(t)$ is the adsorbed amount at time $t$, and $n$ stands for the subscripts 1, 2, and 3. This simple model could be expanded to include parameters accounting for protein denaturation and lateral interactions between adsorbed proteins, however, such parameters were experimentally inaccessible in the present study.

One of the difficulties in modeling protein adsorption is the accurate computation of $c(0, t)$, protein concentration right next to the adsorbing surface at time $t$. Depending on the balance between the rate of arrival of protein molecules to the adsorbing surface and the rate of actual binding to the surface, the progress of adsorption can either be limited by transport, or by actual adsorption to the surface site. The rate of protein molecules arrival to the surface will depend on the manner by which protein is transported from solution to the interfacial region. From a quiescent solution protein will reach the surface by pure diffusion, while from a flowing (or stirred) solution protein will arrive to the interface by a combination of convective and diffusive processes. $^{29}$ Here we consider adsorption of proteins

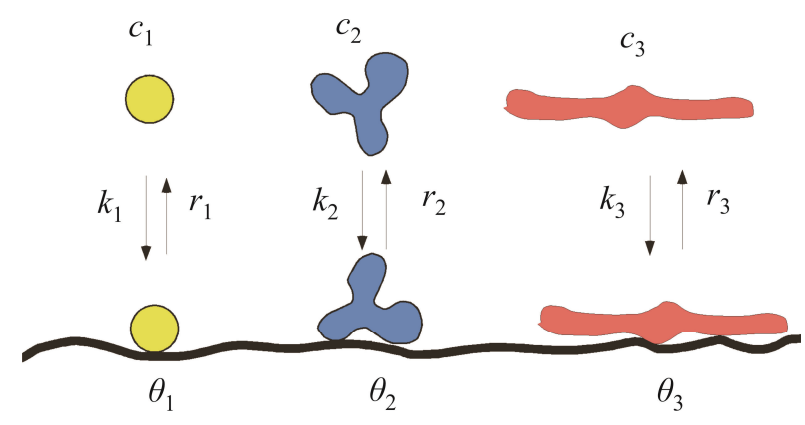

Figure 1. A schematic showing three proteins competitively adsorbing onto a surface.

from a solution that is flowing through a flow channel with rectangular cross-section. If the walls of the flow channel act as sinks for the arriving protein molecules, the flux of protein molecules to the surface ${ }^{2}, J_{n}$, is:

$$
J_{n}=[\Gamma(4 / 3)]^{-1} 9^{-1 / 3}\left(6 q b^{-2} w^{-1} l^{-1} D_{n}^{-1}\right)^{1 / 3} D_{n} c_{n}
$$

where $D_{n}$ is the diffusion coefficient of protein, $c_{n}$ is bulk protein solution concentration, $[\Gamma(4 / 3)]$ is the gamma function of $4 / 3, l$ is the distance along the rectangular flow channel measured from its entrance, $b$ is the thickness of the flow channel, $w$ is the width of the flow channel, and $q$ is the volume flow rate of protein solution. In the absence of desorption $J_{n}$ equals to the adsorption rate $\mathrm{d} \Gamma_{n}(t) / \mathrm{d} t$ and the process is purely transport-limited. In such a case protein surface concentration increases linearly with time and the rate of transport-limited adsorption is proportional to $D_{n}{ }^{2 / 3}, c_{n}$, and to the wall shear rate, $\gamma^{1 / 3}$, which is defined as:

$$
\gamma=6 q b^{-2} w^{-1}
$$

The consequence of the pure transport-limited adsorption is that no intrinsic adsorption-desorption rate constants and protein-surface affinity are experimentally accessible. However, protein adsorption rate is often slower than the flux of protein molecules to the surface and as a consequence, $c(0, t)$ changes during the time of adsorption. When the majority of the surface sites are occupied by the protein(s), the adsorption rate will approach the adsorption-limited rate. The computation of $c(0, t)$ is helped by the concept of the concentration boundary layer (or, so-called "unstirred" layer). ${ }^{31}$ In the case of laminar flow through a rectangular flow channel the thickness of such layer depends on the wall shear rate, protein diffusivity and the distance from the channel entrance. The unstirred layer thickness, $\delta_{n}$, is different for each protein and can be computed from:

\footnotetext{
2 The equivalent heat-transfer problem has been solved by A. Leveque in $1928 .^{30}$
} 


$$
\delta_{n} \approx\left(D_{n} l \mathrm{~g}^{-1}\right)^{1 / 3}
$$

It has been shown that approximately linear protein concentration profile across the concentration boundary layer develops rather rapidly. ${ }^{32}$ Corsel et al. have shown that the adsorption rate equation (equivalent to the eqs. 1) can be solved with the following two boundary conditions: a) the rate of adsorption is linearly dependent to the protein concentration gradient right next to the surface, and b) protein concentration at distances $\delta_{n}$ or greater from the surface equals the protein solution concentration. ${ }^{32}$ The assumption of a linear concentration gradient between the surface and the bulk of the flowing solution leads to a simple expression for $c(0, t)$ :

$$
c_{n}(0, \mathrm{t})=c_{n}-\left(\delta_{n} D_{n}^{-1}\right) \mathrm{d} \Gamma_{n}(t) / \mathrm{d} t
$$

so that the Eq. 1 can be re-cast as:

$$
\begin{aligned}
& \mathrm{d}\left[\Gamma_{1}(t) / \Gamma_{1}^{\max }\right] / \mathrm{d} t= \\
& k_{1}^{\text {app }}\left\{\begin{array}{c}
1-\left[\Gamma_{1}(t) / \Gamma_{1}^{\max }\right] \\
-\left[\Gamma_{2}(t) / \Gamma_{2}^{\max }\right] \\
-\left[\Gamma_{3}(t) / \Gamma_{3}^{\text {max }}\right]
\end{array}\right\} c_{1}-r_{1}^{\text {app }}\left[\Gamma_{1}(t) / \Gamma_{1}^{\max }\right] \\
& \mathrm{d}\left[\Gamma_{2}(t) / \Gamma_{2}^{\max }\right] / \mathrm{d} t= \\
& k_{2}^{\text {app }}\left\{\begin{array}{c}
1-\left[\Gamma_{1}(t) / \Gamma_{1}^{\max }\right] \\
-\left[\Gamma_{2}(t) / \Gamma_{2}{ }^{\max }\right] \\
-\left[\Gamma_{3}(t) / \Gamma_{3}{ }^{\max }\right]
\end{array}\right\} c_{2}-r_{2}^{\text {app }}\left[\Gamma_{2}(t) / \Gamma_{2}{ }^{\max }\right] \\
& \mathrm{d}\left[\Gamma_{3}(t) / \Gamma_{3}^{\max }\right] / \mathrm{d} t= \\
& k_{3}^{\text {app }}\left\{\begin{array}{c}
1-\left[\Gamma_{1}(t) / \Gamma_{1}^{\max }\right] \\
-\left[\Gamma_{2}(t) / \Gamma_{2}^{\max }\right] \\
-\left[\Gamma_{3}(t) / \Gamma_{3}{ }^{\max }\right]
\end{array}\right\} c_{3}-r_{3}^{\text {app }}\left[\Gamma_{3}(t) / \Gamma_{3}{ }^{\max }\right]
\end{aligned}
$$

where $k_{n}^{\text {app }}$ and $r_{n}^{\text {app }}$ are defined as the apparent adsorption rate and desorption rate constants for each of the three proteins:

$$
\begin{aligned}
& k_{n}^{\text {app }}=k_{n} D_{n} /\left(D_{n}+\delta_{n} k_{n}\left[\Gamma_{n}^{\max }-\Gamma_{n}(t)\right]\right) \\
& r_{n}^{\text {app }}=r_{n} D_{n} /\left(D_{n}+\delta_{n} k_{n}\left[\Gamma_{n}^{\max }-\Gamma_{n}(t)\right]\right)
\end{aligned}
$$

The Eqs. 6-10 describe the competition of three proteins for the available surface. This set of equations can be numerically solved and compared with the experimental data, thus providing an experimental access to the adsorption and desorption rate constants.

\section{MATERIALS AND METHODS}

\section{Materials}

3-mercaptopropyltrimethoxysilane (MTS) was from the United Chemical Technologies and was used fresh. Chloramine-T, sodium metabisulfite, sulfuric acid, and hydrogen peroxide were from Sigma. All other solvents used were ACS grade or better. Glassware were cleaned by overnight soaking in sulfuric acid plus Nochromix ${ }^{\text {TM }}$ (Godax Labs) bath, followed by a thorough rising with double-distilled deionized water, and drying in an oven. Human HSA, IgG and Fgn were from Calbiochem. AlexaFluor $^{\circledR} 488$ carboxylic acid, succinimidyl ester (mixed isomers) was from Molecular Probes. $\mathrm{Na}^{125} \mathrm{I}$ was from PerkinElmer.

\section{Surface Gradient Preparation}

The sulfhydryl-to-sulfonate gradient surfaces were prepared on fused silica slides $\left(2.54 \times 7.62 \times 0.1 \mathrm{~cm}^{3}\right.$, ESCO Products) following the methods published earlier. ${ }^{27}$ Silica slides were pre-cleaned using detergent, sonication, and overnight "piranha" bath $\left(v\left(\mathrm{H}_{2} \mathrm{SO}_{4}\right): v\left(\mathrm{H}_{2} \mathrm{O}_{2}\right)=\right.$ 7:3) wash. These substrates were further activated in a three-step process: a 10 minutes exposure to oxygen plasma (Plasmod, Tegal Inc., 50 W@200 mTorr) followed by a 2 hours "piranha" bath and a second 10 minutes of oxygen plasma treatment. Thus activated, the slides were immediately immersed in $1 \%$ MTS solution in toluene for four hours under the dark at room temperature. After the reaction, the slides were sequentially rinsed by toluene, acetone, ethanol, deionized water, and finally dried with dry, filtered nitrogen.

Freshly prepared slide with uniform monolayer of MTS was exposed to UV in ambient atmosphere using a custom-designed motorized set-up to create a sulfhydrylto-sulfonate gradient. The UV source, a low pressure $\mathrm{Hg}$ lamp (Model ELC 4000, Electrolite Corp.) irradiated the slides while an edge mask was moved by motor-controlled linear translator to expose the surface. The edge mask moved at a constant rate of $0.5 \mathrm{~cm} \mathrm{~min}^{-1}$ across center of the slide for 2 minutes, thus creating a $1 \mathrm{~cm}$ long linear oxidation gradient on the center of the slide (Figure 2). On one side of the gradient region was $3 \mathrm{~cm}$ long uniform oxidized MTS region exposed to UV for two minutes, and on the other side was $3 \mathrm{~cm}$ long uniform MTS region that was protected from UV oxidization by the edge mask thus retaining the original surface sulfhydryl groups.

\section{Contact Angle Measurements}

From each batch of gradient surfaces, one silica slide was used to conduct water contact angle measurements. A series of twenty $5 \mu \mathrm{l}$ droplets of double-distilled water were placed along the gradient surface and their contact angles measured using a video goniometer (Cam100, KSV). 
UV radiation
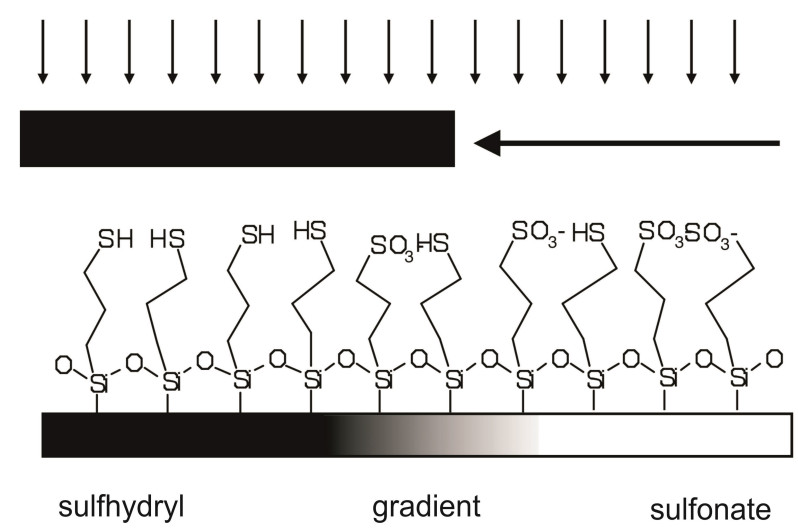

Figure 2. Schematic of sulfhydryl-to-sulfonate gradient preparation method using UV irradiation and a moving mask.

\section{XPS Analysis}

The samples for XPS and SEM analyses were prepared following the same protocols as gradient surfaces except by using smaller fused silica plates $\left(1.0 \times 1.0 \times 0.1 \mathrm{~cm}^{3}\right)$. The samples included uniform monolayers of MTS exposed to UV for 0,1 , and 2 minutes. The XPS spectra were recorded with an XPS spectrometer (Axis-Ultra DLD, Kratos) at the University of Utah Nanofab Lab. The spatial distribution of negative surface charges on the gradient surfaces was analyzed using cationic colloidal gold nanoparticles (average diameter $15 \mathrm{~nm}, \mathrm{SPI}$ ) as adhesion probes. The suspension of cationic gold particles was diluted to $4 \times 10^{9}$ particles $\mathrm{ml}^{-1}$ with phosphate buffer saline (PBS) solution before use, incubated with MTS samples for two hours, rinsed with PBS, double-distilled deionized water, and dried in air. The samples were imaged using high resolution SEM (FEI NovaNano FEG SEM 630) at 40,000× magnification.

\section{Labeling of Proteins}

HSA, IgG or Fgn were each covalently labeled with AlexaFluor488 dye following the protocol from Molecular Probes. ${ }^{33}$ After labeling the protein concentrations were measured using the Biorad ${ }^{\circledR}$ protein assay. The labeling degree of HSA, IgG, and Fgn, defined by a molar ratio of AlexaFluor488 dye to the protein, determined from absorbances at $280 \mathrm{~nm}$ and $460 \mathrm{~nm}$, was found to be $0.78,1.40$, and 0.68 , respectively. Chloramine-T method was used to radio-iodinate HSA, IgG and Fgn with ${ }^{125} \mathrm{I} \cdot{ }^{13}$ A PD-10 column (GE Healthcare) was used to remove the unreacted $\mathrm{Na}^{125}$ I from the protein. The ${ }^{125}$ I-labeled proteins were used within a week.

\section{TIRF Experiments}

A spatially-resolved TIRF technique was utilized to record protein adsorption kinetics. ${ }^{19}$ The TIRF flow cell contained two identical rectangular flow channels allowing for two adsorption experiments to be carried out on a single gradient surface sample. Each adsorption experi- ment was carried out using the solution containing all three proteins at $1 / 100$ of their respective plasma concentrations (i.e. $0.4 \mathrm{mg} \mathrm{ml}^{-1} \mathrm{HSA}+0.12 \mathrm{mg} \mathrm{ml}^{-1} \mathrm{IgG},+$ $0.03 \mathrm{mg} \mathrm{ml}^{-1}$ Fgn) in PBS, $\mathrm{pH} 7.4$, with one of the three proteins being labeled with AlexaFluor488 dye. In each experiment, a ternary protein solution mixture was first flowed for 11 minutes through the channel at a flow rate of $0.70 \mathrm{~cm}^{3} \mathrm{~min}^{-1}$ to replace pre-filled PBS solution and initiate adsorption; after that, PBS solution was injected at the same flow rate for 11 minutes to replace protein solution and initiate desorption. All solutions were flowed in the direction from the oxidized MTS end across the gradient region to the non-oxidized MTS end, i.e., from sulfonate to sulfhydryl regions across the gradient. An evanescent wave created by spatially filtered, expanded $\mathrm{Ar}^{+}$-ion laser beam $(488 \mathrm{~nm}, 0.5 \mathrm{~mW}$ total power) was used to excite fluorescence from adsorbed proteins. The fluorescence emitted along the gradient was imaged using a $50 \mathrm{~mm}$ lens ( $f / 4$, Pentax) onto the slit of a monochromator (1681C, SPEX Industries Inc., $f / 4,2$ $\mathrm{mm}$ slit, 300 grooves $\mathrm{mm}^{-1}$ grating), and recorded every second by a thermo-cooled charged couple device camera (C200, Photometrics). ${ }^{19}$ The fluorescence was background-subtracted and corrected for the laser beam intensity profile. The TIRF adsorption-desorption kinetics were quantified using autoradiography.

\section{Autoradiography Experiments}

In autoradiography experiments, protein adsorption was carried out under the conditions identical to the TIRF experiments, except that the AlexaFluor488-labeled protein was replaced with ${ }^{125} \mathrm{I}$ labeled protein. ${ }^{34}$ After completing the adsorption-desorption cycles, $3 \mathrm{ml}$ of $0.6 \%$ glutaraldehyde in PBS solution were injected into the channel to fix the adsorbed protein molecules for 5 minutes. The channel was dried under nitrogen flow, and the flow cell was disassembled. On a separate uniform MTS plate a set of droplets with known amounts of ${ }^{125}$ I-labeled protein were dried to create a calibration plate for each protein. ${ }^{35}$ The gradient surface with adsorbed ${ }^{125}$ I-labeled proteins and the calibration plate were placed in a plastic bag, brought into contact with a Kodak MS film in a light-tight cassette, and exposed for around 30 hours at $-70{ }^{\circ} \mathrm{C}$. The exposed film was processed in an automated developer system, digitized using a high resolution 16-bit scanner, and analyzed using imaging analysis software (ImageJ, W. Rasband).

\section{RESULTS}

\section{Gradient characterization}

Figure 3 shows the results of surface gradient characterizations. Water contact angles (Figure 3A) decreased smoothly across the gradient region from $62 \pm 3^{\circ}$ (MTS monolayer that was blocked from UV exposure) to $\approx 27$ 

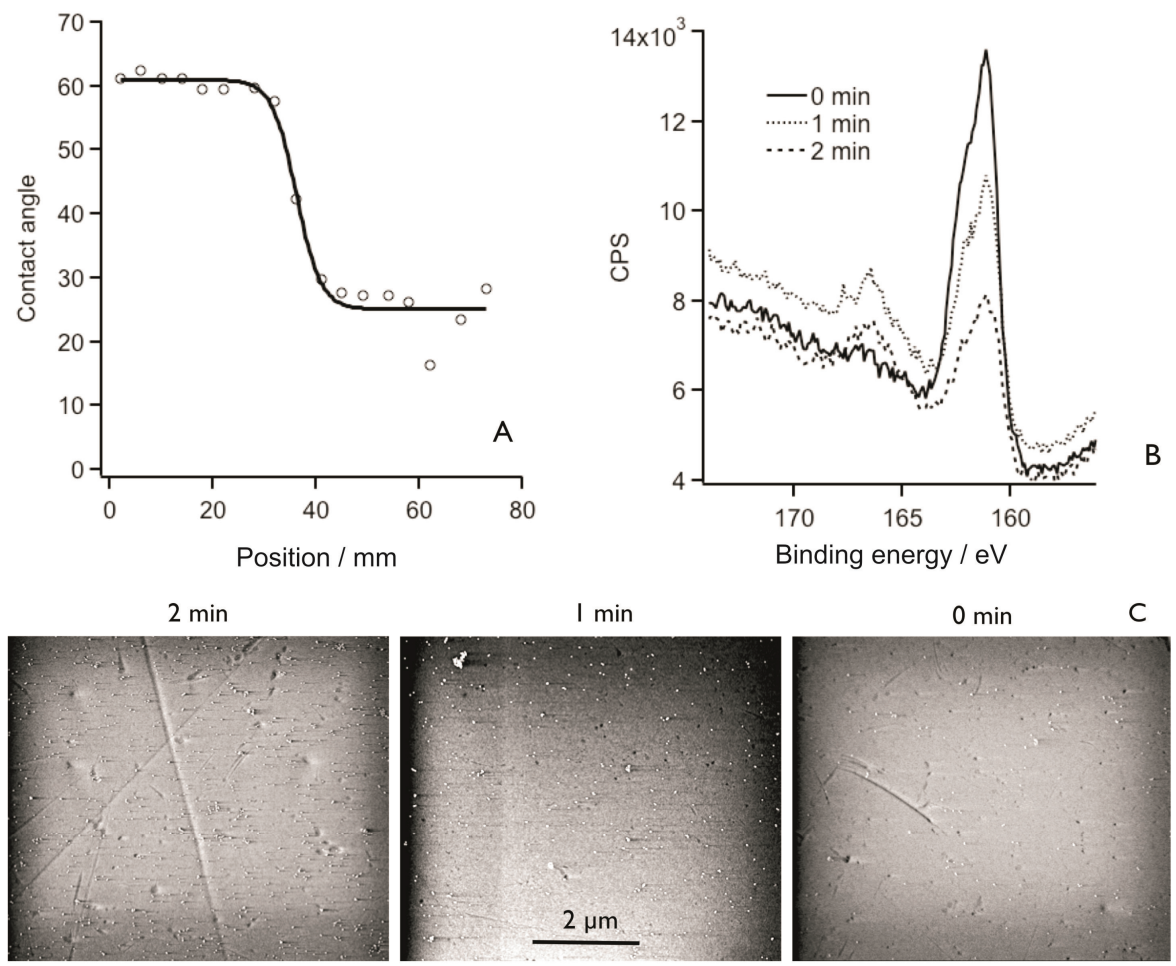

Figure 3. A) Typical water contact angles along the sulfhydryl-to-sulfonate gradient surface prepared with 2 minutes of UV irradiation. B) XPS sulfur S2p spectra of the MTS monolayers after different UV irradiation times. C) SEM images of cationic gold nanoparticles adhered to the MTS monolayers exposed to UV irradiation $(\mathrm{bar}=2 \mu \mathrm{m})$.

$\pm 6^{\circ}$ (MTS monolayer exposed to UV irradiation for 2 minutes). The XPS sulfur S2p spectra (Figure 3B) of the MTS monolayer after different UV irradiation times showed the emergence of a higher binding energy peak at $168 \mathrm{eV}$, indicating the conversion of surface sulfhydryls into sulfonates. The ratio of sulfur in the oxidized state (168 eV peak area) to the non-oxidized state (163.5 $\mathrm{eV}$ peak area) was approx. 30:70 for 2 minutes of UV irradiation, indicating that only about $30 \%$ of surface -SH groups were oxidized by UV. The XPS survey data showed $\approx 2$ atomic $\%$ of sulfur, consistent with previously published data for MTS monolayers. ${ }^{28}$ The uniformity of the MTS monolayer was previously established by mapping the adhesion (pull-off) forces on unmodified and oxidized MTS ${ }^{28}$. The number of cationic gold nanoparticles adhered to the MTS monolayers
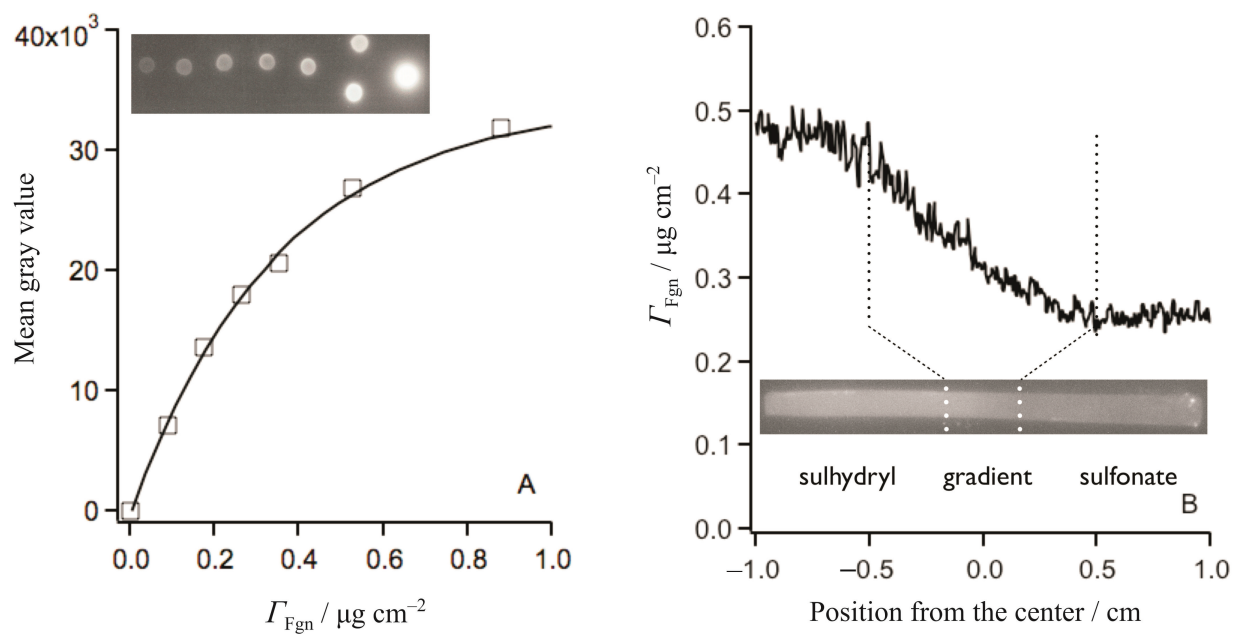

Figure 4. A) Autoradiography calibration plot for ${ }^{125} \mathrm{I}-\mathrm{Fgn}$ (bottom) and the image of the ${ }^{125}$ I-Fgn calibration plate (top). B) ${ }^{125} \mathrm{I}-$ Fgn adsorption profile across the sulfhydryl-to-sulfonate surface gradient. Also shown is the autoradiograph image of the whole flow channel. 

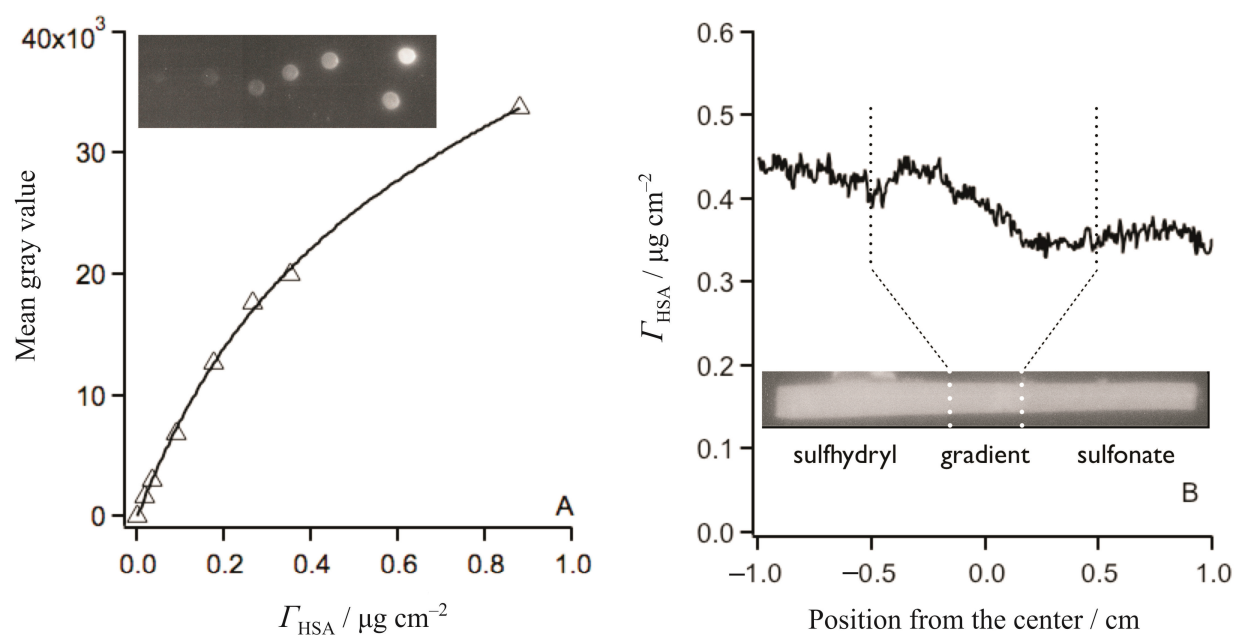

Figure 5. A) Autoradiography calibration plot for ${ }^{125} \mathrm{I}$-HSA (bottom) and the image of the ${ }^{125}$ I-HSA calibration plate (top). B) ${ }_{125}$ I-HSA adsorption profile across the sulfhydryl-to-sulfonate surface gradient. Also shown is the autoradiograph image of the whole flow channel.
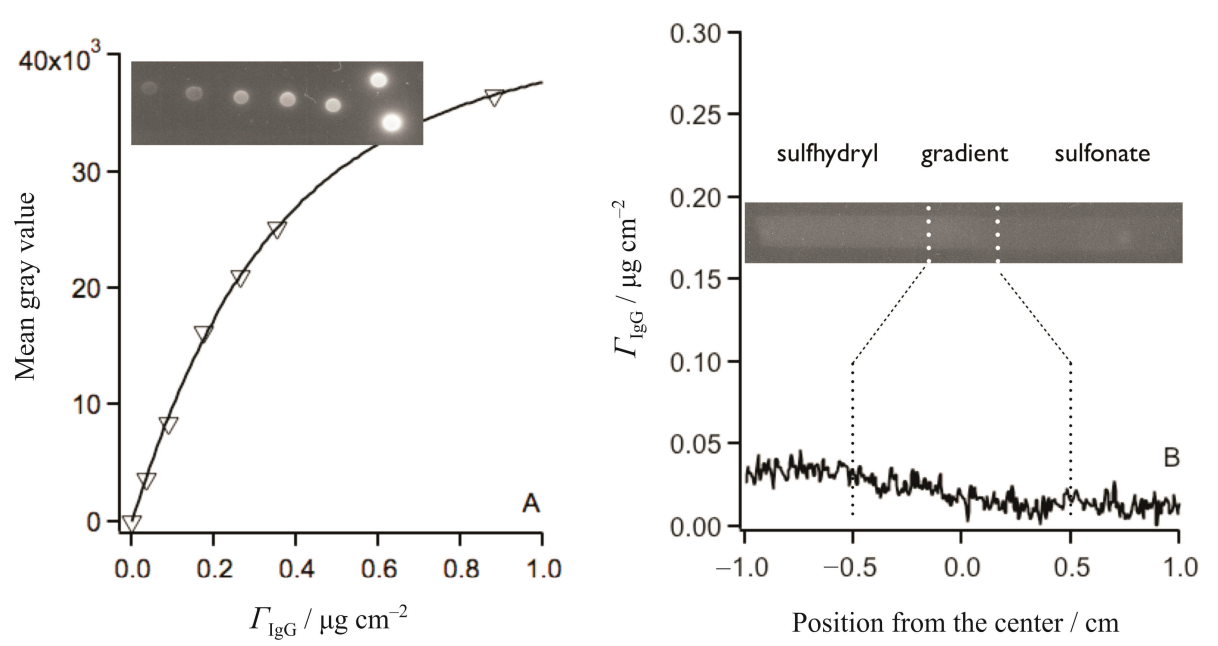

Figure 6. A) Autoradiography calibration plot for ${ }^{125} \mathrm{I}-\mathrm{IgG}$ (bottom) and the image of the ${ }^{125} \mathrm{I}-\mathrm{IgG}$ calibration plate (top). B) ${ }^{125} \mathrm{I}-$ IgG adsorption profile across the sulfhydryl-to-sulfonate surface gradient. Also shown is the autoradiograph image of the whole flow channel.

increased with the UV irradiation times as expected for the conversion of the weakly charged surface sulhydryl groups into the strongly charged sulfonate groups. The average number of particles per $\mu \mathrm{m}^{2}( \pm$ st. dev.) was: $2.6 \pm 1.0$ (no UV), $6.5 \pm 1.5$ ( 1 minute UV) and $18.7 \pm$ 2.9 (2 minutes UV) (Figure 3C).

\section{Autoradiography}

Adsorption profiles recorded by autoradiography after completed adsorption - desorption cycles are shown in Figures 4-6. The left panels show the calibration plots and the autoradiography images of the calibration plates. The right panels show the expanded profile of the protein surface density across the gradient regions and the autoradiography images of the adsorption flow channel.
Adsorption of ${ }^{125} \mathrm{I}$-Fgn from the ternary solution mixture with unlabeled HSA and IgG onto the sulfhydryl-tosulfonate surface gradient is shown in Figure 4.

${ }^{125} \mathrm{I}$-Fgn showed twice as much adsorption $\left(\Gamma_{\mathrm{Fgn}} \approx\right.$ $\left.0.48 \mu \mathrm{g} \mathrm{cm}^{-2}\right)$ on the sulfhydryl end than on the sulfonate end $\left(\Gamma_{\mathrm{Fgn}} \approx 0.25 \mu \mathrm{g} \mathrm{cm}^{-2}\right)$ of the gradient. Adsorption of ${ }^{125}$ I-HSA from the ternary solution mixture with unlabeled Fgn and IgG onto the sulfhydryl-to-sulfonate surface gradient is shown in Figure 5. A smaller adsorption contrast was found across the gradient: $\Gamma_{\mathrm{HSA}} \approx 0.43 \mu \mathrm{g} \mathrm{cm}^{-2}$ on sulfhydryl end vs. $\Gamma_{\mathrm{HSA}} \approx 0.35 \mu \mathrm{g} \mathrm{cm}{ }^{-2}$ on the sulfonate end of the gradient. ${ }^{125} \mathrm{I}$-IgG binding to the sulfhydryl-tosulfonate gradient surface from the ternary solution mixture with unlabeled Fgn and HSA showed ten-fold less adsorption than what was found for Fgn or HSA (Figure 6). 

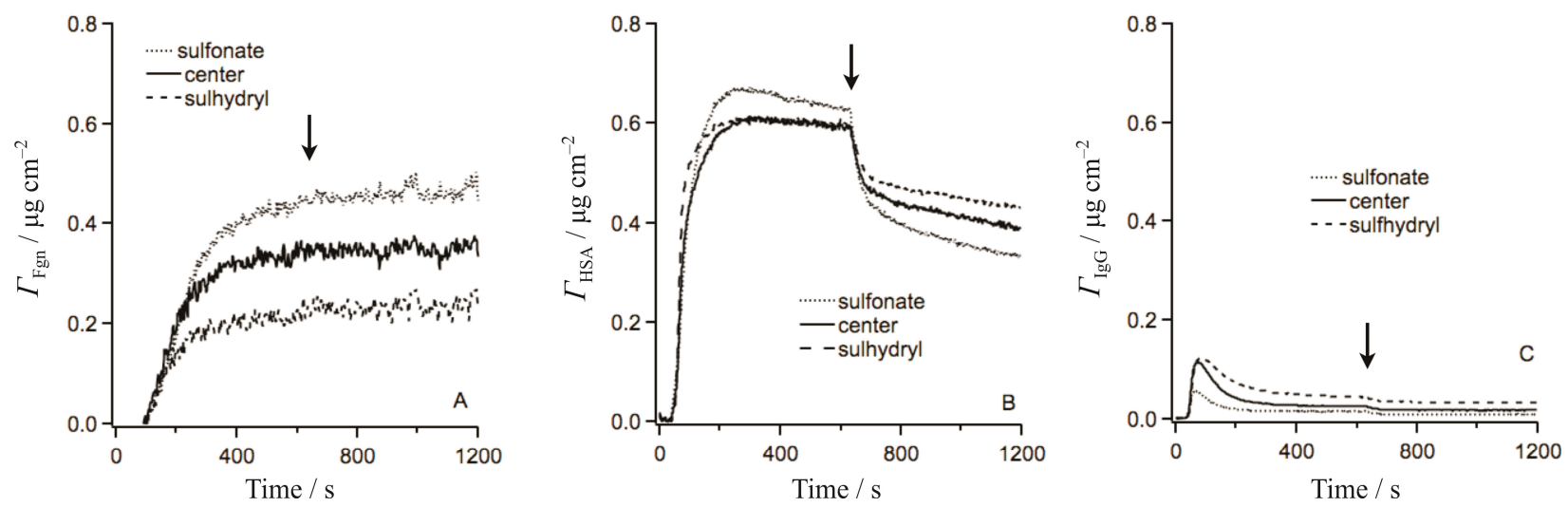

Figure 7. Adsorption-desorption kinetics of AlexaFluor488-labeled Fgn (A), HSA (B) and $\operatorname{IgG}(\mathrm{C})$ from the ternary mixtures onto the sulfonate end, the center, and on the sulfhydryl end of the gradient. Vertical arrows mark the start of the desorption cycles with flow of PBS.

\section{TIRF-measured Adsorption-desorption Kinetics}

Figure 7 shows the TIRF-measured adsorption-desor-ption kinetics of AlexaFluor488-labeled proteins from the ternary solution mixtures after the fluorescence was converted into adsorbed amount using the autoradiography data. The adsorption kinetics on three representative positions are shown: on the sulfonate end, the center of gradient, and on the sulfhydryl end of the gradient, res-pectively. Vertical arrows mark the start of desorption cycles with PBS solution flow starting at $t=660 \mathrm{~s}$. Alexa-Fluor488-Fgn displayed similar initial adsorption rate on each of the three different surface locations $\left(\mathrm{d} \Gamma_{\mathrm{Fgn}} / \mathrm{d} t \approx 1.3 \times 10^{-3} \mu \mathrm{g} \mathrm{cm}{ }^{-2}\right.$ $\mathrm{s}^{-1}$ ) (Figure 7A). The PBS flow did not desorb any adsorbed fibrinogen. AlexaFluor488-HSA showed an early inflexion in the initial adsorption; its highest adsorption rate was found at the downstream sulfhydryl region of the gradient $\left(\mathrm{d} \Gamma_{\mathrm{HSA}} / \mathrm{d} t \approx 1.1 \times 10^{-2} \mu \mathrm{g} \mathrm{cm}^{-2} \mathrm{~s}^{-1}\right)$ (Figure $7 \mathrm{~B}$ ). The HSA adsorption rates were lower at the sulfonate side and at the center of the gradient positions $\left(\mathrm{d} \Gamma_{\mathrm{HSA}} / \mathrm{d} t \approx\right.$ $8.0 \times 10^{-3} \mu \mathrm{g} \mathrm{cm}^{-2} \mathrm{~s}^{-1}$ ), both of which were upstream relative to the sulfhydryl position. A significant amount of desorption of adsorbed Alexa 488-HSA was found with the initial desorption rate of $\mathrm{d} \Gamma_{\mathrm{HSA}} / \mathrm{d} t \approx-2.7 \times 10^{-2} \mu \mathrm{g} \mathrm{cm}^{-2} \mathrm{~s}^{-1}$ at all three gradient positions. Figure $7 \mathrm{C}$ shows the adsorption-desorption kinetics of AlexaFluor488-labeled IgG from the ternary solution mixture. At each of the three gradient positions IgG displayed similar initial adsorption rates $\left(\mathrm{d} \Gamma_{\mathrm{IgG}} / \mathrm{d} t \approx 3.4 \times 10^{-3} \mu \mathrm{g} \mathrm{cm}^{-2} \mathrm{~s}^{-1}\right)$. However, approximately $1-1.5$ minutes after the start of the flow of protein solution a maximum in $\mathrm{IgG}$ adsorbed amount was reached, after which IgG adsorption decreased to a lower, steady state level before the beginning of the PBS-initiated desorption. The transient adsorption maxima of AlexaFluor488-labeled $\mathrm{IgG}$ were found at $\Gamma_{\mathrm{IgG}}=0.124,0.117$, and $0.057 \mu \mathrm{g} \mathrm{cm}^{-2}$ at the sulfhydryl end, the center, and the sulfonate end of the gradient, respectively. PBS desorption further removed about $1 / 3$ of adsorbed AlexaFluor488labeled $\operatorname{IgG}$.

\section{DISCUSSION}

Blood plasma proteins make $7 \%$ of total blood volume. A dozen blood plasma proteins have concentration larger than $1 \mathrm{~g} \mathrm{l}^{-1}$ (Ref. 5). To investigate competitive adsorption of dozen or more proteins on biomaterials from a multi-protein solution mixture could be challenging. In this study we selected the three major proteins: HSA, Fgn, and IgG, for a competitive adsorption on a model gradient surface with a variable surface chemistry. The selected three major proteins are not only the proteins with the high concentration in blood plasma, but are also important in the physiological response to implanted biomaterials. Adsorption of fibrinogen to biomaterials is known to result in platelet adhesion and activation $^{36-38}$ indicating that if fibrinogen is adsorbed to a surface, inactive platelet integrins will bind to it leading to platelet adhesion and activation. ${ }^{39,40}$ Adsorbed HSA has been known to make biomaterial transiently more compatible ${ }^{41,42}$ and suppress the blood platelets activation, ${ }^{43}$ although a recent report claimed otherwise. ${ }^{44} \mathrm{IgG}$ has it's own role in the activation of complement system ${ }^{45}$ and is commonly used in biosensing devices. ${ }^{46}$

In the previous study we estimated that the sulfhydryl-to-sulfonate gradient surface is negatively charged at $\mathrm{pH} 7.4: \approx 0.007 \mathrm{C} / \mathrm{m}^{2}$ at the sulhydryl region and $\approx 0.030$ $\mathrm{C} / \mathrm{m}^{2}$ at the sulfonate region. ${ }^{28}$ The UV irradiation of the MTS monolayer has thus generated both the gradient of surface wettability (Figure 3A) and the gradient of surface charge, ranging from weakly charged sulfhydryl groups to a mixture of strongly charged sulfonates $(\approx 0.3$ surface fraction) and sulfhydryls $(\approx 0.7$ surface fraction). The surface charge densities on both sides of the gradient are insufficient to induce counterion condensation. The gradient of negative surface charge was further confirmed by the adhesion of cationic gold nanoparticles (Figure 3C). In terms of biological relevance the free sulfhydryl groups are capable of forming disulfide bonds with 


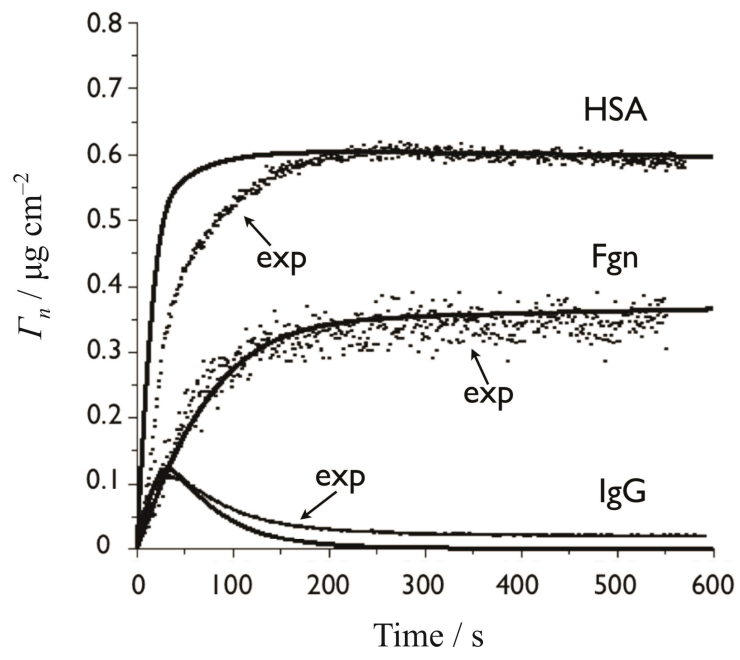

Figure 8. The best overall fit for the competitive adsorption of Fgn, HSA and IgG onto the center of the gradient region. The fitted kinetics are shown in full lines, the experimental data points ("exp") are shown as indicated.

cystein's side chain in proteins, and sulfonate groups are found in many natural protein binding macromolecules such as heparin and glycosaminoglycans.

The adsorption-desorption kinetics can provide important information about protein-surface interactions. For non-uniform surfaces such as surface gradients, the spatially-resolved TIRF technique has been a reliable tool to study the protein adsorption kinetics and establish adsorption and desorption rate constants. ${ }^{47}$ To interpret experimental data in this study we have selected a simple model for protein adsorption from a ternary mixture; a model that assumes competitive adsorption of proteins into a monolayer and neglects eventual protein denaturation and protein-protein lateral interactions, but accounts for the transport of the protein molecules across the concentration boundary layer. The modeling started by finding the desorption rate constant for each protein, $r_{n}{ }^{\text {app }}$, from the desorption cycles of TIRF experiments. Because $c_{n}=0$ during the desorption part of the experiment, Eqs. $6-8$ can be re-written as:

$$
\mathrm{d}\left[\Gamma_{n}(t) / \Gamma_{n}{ }^{\max }\right] / \mathrm{d} t=-r_{n}^{\mathrm{app}}\left[\Gamma_{n}(t) / \Gamma_{n}{ }^{\max }\right] .
$$

At high protein surface coverage when $\left[\Gamma_{n}^{\max }-\Gamma_{n}(t)\right] \rightarrow 0$ there are no free binding sites available for re-adsorption of desorbed molecules so that $r_{n}{ }^{\text {app }} \approx r_{n}$. Once the desorption rates were found for each protein, the approximate thicknesses of the concentration boundary layer were calculated for each protein using eq. $4, \Gamma_{n}^{\max }$ values were taken from the single protein experiments ${ }^{28}$ or were fitted by the model, and the Eqs. 6-10 were simultaneously fitted to the experimental adsorption data for three proteins with three $k_{n}$ rate constants as adjustable parameters.
The best overall fit for the competitive adsorption cycle of the three proteins onto the center of the gradient region is shown in Figure 8. A very good fit for fibrinogen adsorption from the ternary solution mixture was obtained with $k_{\mathrm{Fgn}}=0.008 \mu \mathrm{g}^{-1} \mathrm{~cm}^{3} \mathrm{~s}^{-1}, r_{\mathrm{Fgn}}=\approx 0.0 \mathrm{~s}^{-1}$, $\Gamma_{\mathrm{Fgn}}{ }^{\max }=1.3 \mu \mathrm{g} \mathrm{cm}^{-2}$ (fitted). The simultaneous fit for HSA $\left(k_{\mathrm{HSA}}=0.0004 \mu \mathrm{g}^{-1} \mathrm{~cm}^{3} \mathrm{~s}^{-1}, r_{\mathrm{HSA}}=\approx 0.00005 \mathrm{~s}^{-1}\right.$, $\Gamma_{\mathrm{HSA}}{ }^{\max }=0.83 \mu \mathrm{g} \mathrm{cm}^{-2}$ ) showed a deviation from the experimental data as the fitted adsorbed amounts were higher than the experimental results in the initial adsorption period. Reducing HSA adsorption rate constant, $k_{\mathrm{HSA}}$, and/or increasing the desorption rate constant, $r_{\mathrm{HSA}}$, did not make the HSA fit any better. It is plausible that there was another process in HSA adsorption that has not been predicted by the simple model. It has been suggested that HSA adsorbs into two distinct populations: one that is irreversible bound and other one that is slower but reversible upon desorption with PBS solution. ${ }^{48}$ Indeed, the HSA desorption kinetics was best fitted with two desorption rate constants, and only the higher one was used in our simple model (Figure 7B). The same simultaneous fit predicted well the transient adsorption maximum of $\operatorname{IgG}\left(k_{\mathrm{IgG}}=0.0009 \mu \mathrm{g}^{-1} \mathrm{~cm}^{3} \mathrm{~s}^{-1}\right.$, $\left.r_{\mathrm{IgG}}=\approx 0.04 \mathrm{~s} \mathrm{~s}^{-1}, \Gamma_{\mathrm{IgG}}^{\max }=0.60 \mu \mathrm{g} \mathrm{cm}^{-2}\right)$. While the model seemed to capture correctly the position and the timing of the transient adsorption maximum, it also predicted adsorbed IgG to be completely displaced by the other two proteins before the onset of the desorption cycle, which has not been found in the experiments. This discrepancy may also indicate the existence of the two bound IgG fractions: a fraction that could be displaced by other two proteins and other fraction that could be displaced by the PBS solution. Clearly, the simple model could be improved, for example, by adding the reversible vs. irreversible protein fraction scenarios for HSA and IgG. However, one could argue that there might be other reasons for the observed discrepancies and that any additional scenarios to be included in expanding the simple model would first need to be confirmed by independent experiments. Even with these discrepancies between the fitted and experimental data, the simple model seemed to have served well as a first step towards predicting competitive protein adsorption.

The overall picture of the competitive adsorption of Fgn, HSA and IgG provided by the simple model can be summarized as follows:

a) fibrinogen adsorbed irreversibly, hence its affinity for the surface was the highest of the three proteins,

b) albumin adsorbed reversibly with the dissociation constant, defined as $K_{\mathrm{d}}=r_{n} / k_{n}, K_{\mathrm{d}-\mathrm{HSA}}=$ $0.125 \mu \mathrm{g} \mathrm{cm}^{-3}$ (or, $\approx 1.87 \mathrm{nmol} \mathrm{dm}^{-3}$ ),

c) immunoglobulin $\mathrm{G}$ adsorbed reversibly with the apparent dissociation constant $K_{\mathrm{d}-\mathrm{IgG}}=44.4$ $\mu \mathrm{g} \mathrm{cm}^{-3}$ (or, $\approx 296.3 \mathrm{nmol} \mathrm{dm}{ }^{-3}$ ), 
d) affinity of each protein to the surface (inverse of the dissociation constant) at the gradient center position ranked as: $1 / K_{\mathrm{d}-\mathrm{Fgn}}>1 / K_{\mathrm{d}-\mathrm{HSA}}$ $>1 / K_{\mathrm{d}-\mathrm{IgG}}$,

e) adsorption of both IgG and Fgn was initially transport-limited, and

f) transient adsorption maxima of IgG signified the time of the transition between the transportand adsorption-limited regimes.

The last point $\mathrm{f}$ ) requires the following commentary: initial IgG adsorption rate increased as long as there was enough of the surface available for adsorption. Once the surface filled up with proteins, the IgG adsorption rate slowed down, reached zero (hence, the adsorption maximum), and then became negative (Figure 7C). Because $\mathrm{IgG}$ had the highest desorption rate constant of all three proteins, its lifetime in the adsorbed state was shorter than the surface lifetime of Fgn or HSA molecules. By being more frequent, IgG desorption events created an opportunity for the other two proteins to bind to the surface sites left open by IgG. As a consequence of this competitive exchange process the adsorbed layer was slowly depleted from the bound IgG molecules. Similar, but much less pronounced transient adsorption maxima could also be seen in HSA adsorption kinetics on the sulfonate side of the gradient (Figure 7B), indicating that HSA desorption rate constant for this surface region was larger than the desorption rate constant of Fgn. The competitive adsorbed protein exchange process, the socalled "Vroman" effect, illustrates the adsorption dynamics of the blood protein - biomaterials interactions. ${ }^{9}$ The composition of the adsorbed proteins layer on biomaterials changes over time and so does the host physiological response to the implanted material.

\section{CONCLUSION}

Competitive adsorption of three major human plasma proteins, albumin, fibrinogen, and immunoglobulin $\mathrm{G}$, illustrates the complex dynamics of blood proteins biomaterials interactions. The three proteins displayed different adsorption and desorption characteristics with each protein adsorbing less to the sulfonate region than to sulfhydryl region of the gradient. The TIRF-measured adsorption-desorption kinetics revealed the differences in the adsorption and desorption of three proteins. By fitting the experimental data to a simple model of competitive protein adsorption, the affinity of each protein to the surface at the gradient center position was ranked as: $1 / K_{\mathrm{d}-\mathrm{Fgn}}>1 / K_{\mathrm{d}-\mathrm{HSA}}>1 / K_{\mathrm{d}-\mathrm{IgG}}$. The competitive exchange of the adsorbed proteins was related to the magnitude of their desorption rate constants. The simple model failed to perfectly fit HSA, and to some degree IgG adsorption data, most likely due to the presence of two adsorbed fractions, reversibly and irreversibly bound, for these two proteins. In contrast, Fgn adsorption data were fitted well as being initially transportlimited and completely irreversible.

Acknowledgements. This study was supported by an NIH grant (R01 HL084586). We thank B. Wright for the preliminary work on developing the protocol for preparation of gradient surfaces.

\section{REFERENCES}

1. B. A. Shinoda and R. G. Mason, Biomat. Med. Dev. Art. Org. 6 (1978) 305-329.

2. R. Lindsay, Trans. Am. Soc. Artif. Int. Organs 26 (1980) 603-610.

3. T. A. Horbett, Cardiovasc. Pathol. 2 (1993) 137S-148S.

4. W. G. Pitt, K. Park, and S. L. Cooper, J. Colloid Interface Sci. 111 (1986) 343-362.

5. J. D. Andrade and V. Hlady, Ann. N. Y. Acad. Sci. 516 (1987) $158-172$.

6. J. M. Anderson, T. L. Bonfield, and N. P. Ziats, Int. J. Artif. Org 13 (1990) 375-382.

7. L. Tang and J. W. Eaton, Am. J. Clin. Path. 103 (1995) 466-471.

8. S. M. Slack and T. A. Horbett, J. Colloid Interface Sci. 133 (1989) 148-165.

9. E. F. Leonard, V. T. Turrito, and L. Vroman (Eds), Blood in Contact with Natural and Artificial Surfaces. Vol. 516, N.Y. Acad. Sci., New York, 1987.

10. J. L. Brash and P. ten Hove, J. Biomater. Sci. Polym. Ed. 4 (1993) 591-599.

11. J. L. Brash and P. W. Wojciechowski (Eds), Interfacial Behavior of Bioproducts., M. Dekker, New York, 1996.

12. M. Malmsten (Ed.), Biopolymers at Interfaces, $2^{\text {nd }}$ ed., M. Dekker, New York, 2001.

13. V. Hlady, J. Buijs, and H. P. Jennissen, Methods for studying protein adsorption, in: R. Wetzel (Ed.), Amyloid, Prions, and Other Protein Aggregates, Methods Enzymol. 309 (1999) 402-429.

14. J. L. Brash, J. Biomater Sci. Polym. Ed. 11 (2000) 1135-1146.

15. J. Genzer and R. R. Bhat, Langmuir 24 (2008) 2294-2317.

16. S. Morgenthaler, C. Zink, and N. D. Spencer, Soft Matter 4 (2008) 419-434.

17. H. Elwing and C.-G. Gölander, Adv. Colloid Interface Sci. 32 (1990) 317-339

18. H. Elwing, S. Welin, A. Askendal, U. Nilsson, and I. Lundström, J. Colloid Interface Sci. 119 (1987) 203-210.

19. V. Hlady, Appl. Spectrosc. 45 (1991) 246-252.

20. C.-G. Gölander, Y.-S. Lin, V. Hlady, and J. D. Andrade, Colloids Surf. 49 (1990) 289-302.

21. Y.-S. Lin and V. Hlady, Colloids and Surfaces B. Biointerfaces 2 (1994) 481-491.

22. Y.-S. Lin and V. Hlady, Colloids and Surfaces B. Biointerfaces 4 (1995) 65-75.

23. Y.-S. Lin, V. Hlady, and C.-G. Gölander, Colloids and Surfaces B: Biointerfaces 3 (1994) 49-62.

24. V. Hlady and C.-H. Ho, Materialwiss. Werkst. 32 (2001) 185-192.

25. V. Hlady, Adsorption kinetics of low density lipoprotein onto a hydrophobic-hydrophilic gradient surface, in: G. Pifat-Mrzljak (Ed.), Supramolecular Structure and Function 7, Kluwer Academic, New York, 2001, pp. 45-61.

26. H. T. Spijker, R. Bos, W. van Oeveren, J. de Vries, and H. J. Busscher, Colloid Surface B 15 (1999) 89-97.

27. L. E. Corum and V. Hlady, Biomaterials 31 (2010) 3148-3155.

28. Y.-X. Ding, S. Streitmatter, B. E. Wright, and V. Hlady, Langmuir 26 (2010) 12140-12146.

29. B. K. Lok, Y. L. Cheng, and C. R. Robertson, J. Colloid 
Interface Sci. 19 (1983) 104-116.

30. A. Léveque, Annales des Mines XIII (1928) 283.

31. T. M. Squires, R. J. Messinger, and S. R. Manalis, Nat. Biotechnol. 26 (2008) 417-426.

32. J. W. Corsel, G. M, Willems, J. M. M. Kop, P. A. Cuypers, W. T. Hermens, J. Colloid Interface Sci. 111 (1986) 544-554.

33. Molecular Probes. Alexa Fluor Succinimidyl Esters, 6 February 2007.

34. Y.-S. Lin, V. Hlady, and J. Janatova, Biomaterials 13 (1992) 497-504.

35. C.-H. Ho, V. Hlady, G. Nyquist, J. D. Andrade, and K. D. Caldwell, J. Biomed. Mater. Res. 25 (1991) 423-441.

36. J. L. Brash and P. ten Hove, Thromb. Haemost. 51 (1984) 326-330.

37. J. N. Lindon, G. McManama, L. Kushner, E. W. Merrill, and E.W. Saltzman, Blood 68 (1986) 355-362.

38. J. M. Grunkemeier, W. B. Tsai, C. D. McFarland, and T. A. Horbett, Biomaterials 21 (2000) 2243-2252.

39. B. Savage and Z. M. Ruggeri, J. Biol. Chem. 266 (1991) 11227-11233.

40. S. N. Rodrigues, I. C. Goncalves, M. C. L. Martins, M. A Barbosa, and B. D. Ratner, Biomaterials 27 (2006) 5357-5367.

41. R. C. Eberhart, M. S. Munro, J. R. Frautschi, M. Lubin, F. J.
Clubb Jr., C. W. Miller, and V. I. Sevastianov, Ann. N. Y. Acad. Sci. 516 (1987) 78-95.

42. R. C. Eberhart and C. P. Clagett, Semin. Hematol. 28 (1991) $42-48$.

43. K. Kottke-Marchant, J. M. Anderson, Y. Umemura, and R. E. Marchant, Biomaterials 10 (1989) 147-155.

44. B. Sivaraman and R. A. Latour, Biomaterials 31 (2010) 1036-1044.

45. J. M. Anderson, Inflammation, wound healing and foreign body response, in: B. D. Ratner, A. S., Hoffman, F. J. Schoen, and J. E. Lemons (Eds), Biomaterials science, 2nd ed., Elsevier, Amsterdam, 2004, pp 296-304.

46. J. N. Herron, H. K. Wang, V. Janatova, J. D. Durtschi, D. A. Christensen, K. D. Caldwell, I.-N. Chang, and S.-C Huang, Orientation and activity of immobilized antibodies, in: $\mathrm{M}$. Malmsten (Ed.), Biopolymers at interfaces, 2nd ed., M. Dekker, New York, 2000, Vol. 110, pp 115-164.

47. V. Hlady, C.-H. Ho, and D. W. Britt, Quantitative Analysis of Protein Adsorption Kinetics, in: N. Kallay (Ed.), Interfacial Dynamics, M. Dekker, New York, 2000, Vol. 88, pp. 405-418.

48. R. Kurrat, J. E. Prenosil, and J. J. Ramsden, J. Colloid Interface Sci. 185 (1997) 1-8. 